\title{
Ecotone response to climatic variability depends on stress gradient interactions
}

\author{
George P. Malanson', Lynn M. Resler ${ }^{2}$ and Diana F. Tomback ${ }^{3^{*}}$
}

\begin{abstract}
Background: Variability added to directional climate change could have consequences for ecotone community responses, or positive and negative variations could balance. The response will depend on interactions among individuals along environmental gradients, further affected by stress gradient effects.

Methods: Two instantiations of the stress gradient hypothesis, simple stress and a size-mediated model, are represented in a spatially explicit agent based simulation of an ecotone derived from observations of Abies lasiocarpa, Picea engelmannii, and Pinus albicaulis in the northern Rocky Mountains. The simple model has two hierarchically competitive species on a single environmental gradient. The environment undergoes progressive climate change and increases in variability. Because the size model includes system memory, it is expected to buffer the effects of extreme events.

Results: The interactions included in both models of the stress gradient hypothesis similarly reduce the effects of increasing climatic variability. With climate amelioration, the spatial pattern at the ecotone shows an advance of both species into what had been a higher stress area, but with less density when variation increases. In the size-mediated model the competitive species advances farther along the stress gradient at the expense of the second species. The memory embedded in the size-mediated model does not appear to buffer extreme events because the interactions between the two species within their shifting ecotone determine the outcomes.
\end{abstract}

Conclusions: Ecotone responses are determined by the differences in slopes of the species response to the environment near their point of intersection and further changed by whether neighbor interactions are competitive. Interactions are more diverse and more interwoven than previously conceived, and their quantification will be necessary to move beyond simplistic species distribution models.

Keywords: Agent based model, Competition, Ecotone, Extinction, Facilitation, Simulation, Variability

\section{Background}

As the global climate changes, shifts in climatic variability, including extremes, could have ecological consequences at least as great as would result from changes in mean temperature and precipitation [1-3]. Thus, the ecological impacts of climatic variability requires research $[4,5]$, specifically on how climate variability will affect population processes, and how to apply basic ecological theory to understand community responses to climate change. Current theory is rooted in ideas on frequency dependent population processes such as competition [6], but ideas based on facilitation may provide new insights [7] necessitating 1) the need to incorporate interactions among organisms in species distribution models, e.g. [8], and 2) recognition of climatic variation [8]. We aim to examine how different hypothesized modes of plant interaction alter ecological response to changing climatic variability. We use a spatially explicit, two-species, agent-based model (derived from an earlier model of alpine treeline in the Rocky Mountains, USA) with contrasting gradients of change in the relative strength of competition and facilitation to simulate responses of an ecotone to climatic variability.

\section{Climatic variability}

Work on climatic variability has indicated that anomalies (in climatology, the difference from average) in one
${ }^{3}$ Department of Integrative Biology, CB 171, University of Colorado Denver,

P.O. Box 173364, Denver, CO 80217, USA

Full list of author information is available at the end of the article 
direction could be balanced by anomalies in the other direction. However, the potential ecological importance of climatic variability has also been recognized. For example, extreme events change high or low temperature regimes and exacerbate moisture limitations from a state of stress to one of disturbance, because their physiological effects change from reduced productivity to mortality. Kayler et al. have called for inclusion of variation in experimental approaches to ecological response [9]. Introducing variability in rain-exclusion or-addition experiments should be straightforward, but otherwise welldeveloped experiments sometimes do not explore this factor (or have been too short). Where variation has been introduced, experiments show the effects of functional diversity [10]. From simulations, Malanson [11] reported that competition affected population responses to increasing variability more than biological (i.e., genetic) or spatial constraints.

\section{Ecotones and facilitation}

Ecotones have been a focus for examining ecological response to climate change [12]. For treeline ecotones in particular, research has focused on positive interactions among individuals and has downplayed competition [13]. Given the observation of ecotone boundaries that were abrupt relative to the environmental gradient in which they were observed, the positive feedback switch hypothesis [14] became the dominant explanation; e.g., [15-17]. This emphasis on facilitation at ecotones such as alpine treeline became blended with the stress gradient hypothesis (SGH) [18, 19] (which is flexible in the details depending on the stressor; alpine treelines can be locally water stressed within a broader scale stress of heat deficit [13]). de Dios et al. showed how a temporal switch in interaction could be a factor in ecotone response to climate change [20]. Rates of response to climate change may also be tied to species interactions [21]. Moreover, treeline dynamics have been shown to respond to interannual climatic variability [22].

At alpine treeline ecotones the population dynamics and spatial distributions of conifers are affected by both negative and positive feedbacks within their community. The canopy reduces wind, which in turn reduces evapotranspirative stress in its neighborhood, captures snow that becomes late-summer soil moisture in an otherwise dry environment, and increases the accumulation of fine sediment and organic matter in the soil $[13,23]$. Further, the canopy reduces the impact of UV radiation and of night sky exposure and cold temperature photoinhibition in its neighborhood [24]. Conversely, the shade cast by closed canopies (especially in krummholz) limits light availability, and perhaps more importantly, maintains colder soil temperatures under the canopy, which may be the limiting factor for treelines at global scale [25] (high density can also result in competition for soil resources, including water); single trees may have different effects [26]. A variety of interactions are being explored as limits on treeline upslope advance in response to climate change [27].

At treeline, the extreme limit of the stress gradient for trees, the most exposed individuals can benefit most from positive feedback. Moreover, the positive feedbacks at the edge may be critical to tree recruitment, whereas the negative feedbacks may be limited by the small size of individuals ([28]. This size dependence is part of the rationale for our size gradient model, and incorporating relative sizes of interacting plants and the resulting effects more be a more realistic portrayal of community processes.

\section{Rationale}

We use a corollary to the $\mathrm{SGH}$, a relative size gradient (RSG) model [29], which incorporates a more mechanistic feedback between the abiotic and biotic components of the environment. The SGH holds that competition will decrease and facilitation increase along a gradient of increasing environmental stress $[30,31]$ (. The particular environmental processes or gradients through which interactions operate are varied and potentially combined [32]. The RSG model mediates the SGH by providing a more direct mechanism for a change in net interaction between positive and negative and a feedback through growth rates. Malanson and Resler found that the RSG produces similar patterns of response to climate change in simulations [29]; the reason is that in the area of high stress the largest plants are never so much larger than the smaller that they become competitors instead of facilitators.

The response of alpine treeline to increasing variability with climate change could depend on whether the feedback process is a direct function of level of environmental stress (the classic SGH) or mediated by plant size, as we suggest. The SGH embodies a direct effect of plants on the local environment, modifying the stress on other plants. The size gradient model builds memory into the system. The sizes of plants can increase only incrementally (but can decrease drastically with mortality) so the effects of climatic variability are buffered by the existing size of plants. We hypothesize that a stress gradient system will be more sensitive to increasing variation in the climate than would a size gradient system because the feedback within the latter will change slowly. The addition of a size gradient model contributes to an effort to include a variety of plant-plant interactions in the study of species range limits with climate change, e.g., [33].

To examine how interactions mediate variability, we model the ecotone dynamics between two populations by simulating the effects of a changing climate with increasing variability on a spatially explicit environmental gradient. We simulate population dynamics and 
extinctions, which are likely to respond to variability [34]; although demographic stochasticity is included, we do not manipulate it. Ecotones may be revealing indicators of the consequences of climate change. Alpine treelines are simple in that they support few tree species (often 1-2), and the environmental gradient, from the "plant's eye view" (sensu Harper, p 706 [35]), is relatively simple if the decline in tree size and density is assumed to be indicative. We acknowledge that the model is so simplified that it will not differentiate all of the specific processes; cf. [36]. Körner recently emphasized the role of size on feedbacks, but did not lay out a complete framework for the role of size that would capture effects of neighbors and water limitations [28]. Although this is the type of two-species model that is common in theoretical ecology, it is based on observations of Pinus albicaulis, Abies lasiocarpa, and Picea engelmannii. Observations at alpine treeline ecotones in the northern Rocky Mountains indicate that $P$. albicaulis is a pioneer species, able to establish in open areas where it then provides facilitation for the establishment of A. lasiocarpa and $P$. engelmannii [37]. The mechanistic nature of facilitation indicates that size is likely to be relevant [26], and neighbors affect size [38].

We hypothesize that the simulation of outcomes in populations, extinctions, and spatial outcomes will differ among the models of no interaction (none), SGH interaction (stress), and the size model (size) of interaction. Our expectation is that stress gradient and size gradient feedback models will buffer the response to climatic variability so that the relative sensitivity would be: none $>$ stress $>$ size.

\section{Methods}

\section{Conceptual model}

We use a simplistic model to elucidate the effects of variability for the types of environmental gradients and species niche dimensions envisioned for ecotones, specifically for hierarchical competition and the SGH. We use an approach that eliminates the need to know the response of a particular system to specific changes in climate. Instead, we consider the plant's-eye-view by varying habitat quality as a composite parameter and change it through time with and without increasing variability during a period of improving habitat quality.

The model assumes that interactions among plants are based on the relative size of the focal individual and the summed sizes of its neighbors and that this interaction intensity is a multiplier the habitat quality, which is $<1$ for competitive effects and $>1$ for facilitative effects; the range is from 0.5 to 2; [39] for details. The model computes the habitat quality given an initial environmental gradient multiplied by the interaction intensity. This habitat quality is then used to determine the probability of reproduction, the amount of growth, and the probability of mortality.

\section{Model design}

We use a spatially explicit agent based model created in Netlogo [40], Additional file 1. The model is based on a grid of 1000 x 50 cells, wrapped as a cylinder to eliminate edge effects. The plant's-eye-view of the environment is defined by responses of two species $(\mathrm{SpS}$, for specialist and SpG for generalist, subscript $\mathrm{s}$ ) on the long axis of the grid set as hierarchical Gaussian curves, $E_{\mathrm{ys}}, 1-0$ across the rows (subscript y) as shown in Fig. 1 (cf. [41]). This approach collapses the various dimensions of niche to a single gradient appropriate for a simple exploratory model. The responses in this model are relative but are based on observations of Abies lasiocarpa and Picea engelmannii, for SpS, and Pinus albicaulis, for SpG, at alpine treeline in western North America (see [42]). P. albicaulis is a pioneer, keystone, and foundational species at alpine treeline; it is able to reproduce in highstress environments without neighbor facilitation, and it grows slowly. A. lasiocarpa and $P$. engelmanni often establish next to extant $P$. albicaulis and have somewhat higher growth rates. Information on survivorship is sparse because treeline sites are relatively new (since the Little Ice Age in the Rocky Mountains [43]) but the ages of $P$. albicaulis are much greater than any of the other two species at sites where we have examined tree rings (e.g., [44]). Although the gradient is smooth, the feedback that is simulated creates clumped patterns of species occurrences. Feedback is likely to be more important in the spatial heterogeneity of factors such as microclimate or soils than are purely abiotic processes [45].

Each cell of the grid can be occupied by one individual, and to initialize model runs, all cells are occupied by an individual $j$, at a random size between 1 and 100, with a probability proportional to $\mathrm{E}_{\mathrm{ys}}$. The dynamics of the

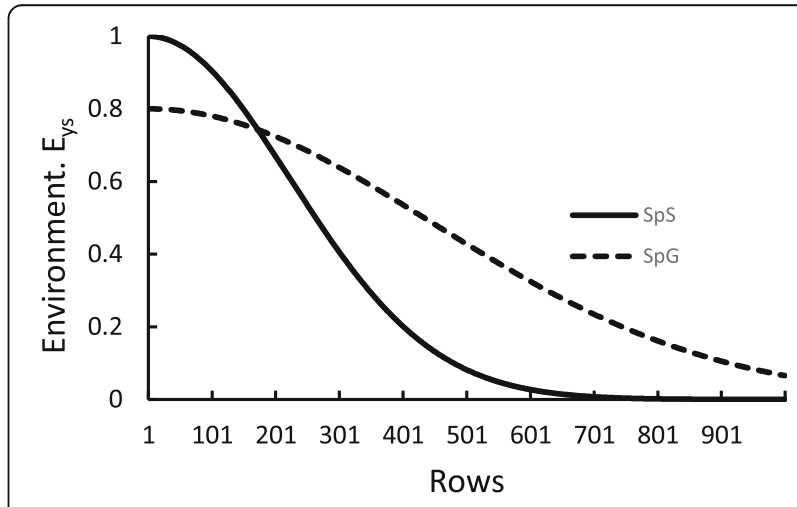

Fig. 1 The species-specific habitat quality assigned to each row of the grid. This value is used in multiplier to compute the probability of recruitment and mortality and the amount of growth 
population are simulated over 400 iterations of recruitment, growth, and death as Monte Carlo processes with treatments implemented in the last 100 iterations. Although the initial size distribution is random rather than realistic, age structure develops during the initial 300 iterations before treatments begin.

The stress gradient version is computed as a logarithmic increase in interaction intensity with the number of neighbors: multiplied by a gradient from 0.5 to 2 across the length of the grid:

$$
\begin{aligned}
& \mathrm{d}=\text { number of neighbors } \\
& \mathrm{I}_{\text {stress }}=.26+.333 \ln \mathrm{d} *\left(2-1.5 \mathrm{E}_{\mathrm{y}}\right)
\end{aligned}
$$

The size gradient version is

$$
\begin{aligned}
& \mathrm{d}=\mathrm{S}_{\mathrm{n}} / \mathrm{S}_{\mathrm{f}} \\
& \mathrm{I}_{\text {size }} \mid \mathrm{d}<33.333=1+.03 \mathrm{~d} \\
& \mathrm{I}_{\text {size }} \mid \mathrm{d}>33.333=3.25-.0375 \mathrm{~d}
\end{aligned}
$$

where $S_{n}$ is the sum of the sizes of the eight neighbors and $S_{\mathrm{f}}$ is the size of the focal individual; for empty cells the intensity for recruitment is calculated with $S_{f}=1$ interaction intensities less than 0.5 or greater than 2 are reset to these limits (Fig. 1). A logarithmic form was chosen to give most weight to the first neighbor; cf. [29, 36]. While it would seem that the model would be sensitive to relative size at which competition begins to outweigh facilitation (here at 10 times, with competition reaching its limit at 30), trials with these points set at 3/ 10 and 33.33/100 produce quantitatively similar results. Although the stress gradient is linear with the environment, spatial patterns develop because of the density of neighbors, especially at the treeline.

Recruitment for SpS and SpG occurs on cells with probability a function of the size of their extant population relative to the size of the grid and the environment of the row:

$$
\begin{aligned}
& \mathrm{P}\left(\mathrm{R}_{1}\right)=\mathrm{rN} \mathrm{IE}_{\mathrm{y} 1} \\
& \mathrm{P}\left(\mathrm{R}_{2}\right)=\mathrm{rN} \mathrm{IE}_{\mathrm{y} 2}
\end{aligned}
$$

where $\mathrm{N}$ is the current population and $\mathrm{r}$ is 0.00001 or 0.000008 based on the size of the grid, so that the maxima would be with $\mathrm{rN}=1.0$ or 0.64 for $\mathrm{SpS}$ and SpG, respectively. SpG can establish only on empty cells whereas $\mathrm{SpS}$ can replace SpG but with its recruitment rate halved. Recruitment parameters are chosen to reduce dimensionality by setting them to allow replacement recruitment in a hypothetical open, ideal environment. Because of the feedbacks, recruitment in the neighborhood of extant individuals is more common in the treeline area.

SpS has a higher mortality rate than SpG to match its higher reproductive rate:

$$
\begin{aligned}
& \mathrm{P}\left(\mathrm{M}_{1}\right)=1-.5 \mathrm{IE}_{\mathrm{y} 1} \\
& \mathrm{P}\left(\mathrm{M}_{2}\right)=1-.4 \mathrm{IE}_{\mathrm{y} 2}
\end{aligned}
$$

The maxima are set at half of the maxima for $\mathrm{E}_{\mathrm{ys}}(1.0$ and 0.8 ) based on the logic that once established mortality is relatively rare.

\section{Model runs}

To represent climate change, the value of $E_{y s}$ is reset to $\mathrm{E}_{\mathrm{y}-1 \mathrm{~s}}$ in iterations 301-400. $\mathrm{E}_{\mathrm{ys}}$ increases; the climate ameliorates in the plant's-eye-view. Iterations 1-300 with $\mathrm{E}_{\mathrm{ys}}$ constant allow all relevant variables to equilibrate.

Climatic variability is created by multiplying the values for all cells in every year by a random number drawn from a normal distribution with a mean of 1 and a standard deviation of 0.25 . We use 0.25 based on the standard deviation of standardized tree-ring widths recorded for a $>400$ year-old stand of alpine larch at treeline in Glacier National Park, which range from 0.22 to 0.25 (personal communication, Greg Pederson); comparison of the two values has revealed much higher extinction rates for 0.25 when the multiplier doubled over 200 year [11]. We increase the multiplier to 1.5 over 100 year, after which it again becomes constant to represent a new climatic equilibrium. With the multiplier $\mathrm{E}_{\mathrm{ys}}$ is constrained to not exceed 1.0 or 0.8 for SpS and SpG, respectively.

We present the results of six simulations, but we show the differences between scenarios with constant and with increasing climatic variability for simulations with no feedback, with the stress gradient feedback, and for the size gradient feedback. The metrics of analysis here are the differences in the population sizes of SpS and SpG between the climatic representations averaged from 100 replicate runs, and the proportion of those runs that end in extinction for either species (extinction/200); simulation runs in which extinction occurred during the first 20 iterations, prior to equilibration of population sizes, were discarded and replaced.

\section{Results}

Even before climate change begins in the simulations, the effect of feedbacks on response to variation becomes evident (Table 1A). In terms of overall population numbers, the feedbacks are able to buffer the population responses to increasing variability. The small difference between the stress and size gradient models depends on the species.

With climate change and increasing variability, however, effects are more pronounced (Table 1B). Rather than clearly buffering the population responses to increasing variability as expected, the simulation representing the size gradient hypothesis had population 
Table 1 Percent difference in each population for the two model species between scenarios with and without climatic variation for a control with no feedback and the stress and relative size gradient models at two times in the simulations runs

\begin{tabular}{llll}
\hline Models: & None & Stress & Size \\
\hline A & & & \\
SpS & -3.5 & 1.0 & -0.4 \\
SpG & -2.6 & -2.0 & -1.0 \\
B & & & \\
SpS & -17 & -26 & -18 \\
SpG & -33 & -11 & -42 \\
\hline
\end{tabular}

A) before climate change (at iteration 300), and B) after climate change (iteration 400)

changes similar to the no feedback scenario especially for $\mathrm{SpS}$, while for $\mathrm{SpG}$ the size gradient version had greater population loss with climate change and increasing variability and the stress gradient version lost least.

The stress gradient hypothesis is able to minimize the impact of variability on SpG by increasing the intensity and effect of facilitation in the area of the grid that was previously empty, but with climate change becomes habitable. However, because competition dominates in the area occupied preferentially by $\mathrm{SpS}$, i.e. the better habitat quality, in the stress gradient simulations, its population is more strongly affected by increasing variability than either the no feedback or size gradient versions. The size gradient hypothesis turns out to be sensitive to variation. In the high stress part of the gradient, the effects of size are limited because growth rates are low and sizes remain small. Relative to the amplitude of climatic variation, the effects of feedback remain small in this area.

In proportional change, the stress gradient representation buffered the responses of the populations more than that of the size gradient hypothesis, contrary to our expectations. However, in absolute terms this difference is because the size gradient maintained larger populations than did the stress gradient as simulated (Fig. 2). With the size gradient the population of $\mathrm{SpS}$ is higher before climate change and both populations are higher after it. While the stress gradient, in theory, could allow individuals to develop larger populations in the high stress portion of the gradient, any facilitation depends on multiple rare recruitments, and with climatic variability rare individuals are eliminated before they are joined by others.

With respect to the probability of extinction, simulations indicated that the stress and size gradient models showed little difference in effect (Table 2). A visualization helps to interpret the model outcomes (Fig. 3). With climate change (B, C, E, F, H, I) the populations advance into the higher stress area. With increasing variability, the populations are not as dense as the simulations with no variability. The advance for $\mathrm{SpS}$ is not as retarded by the

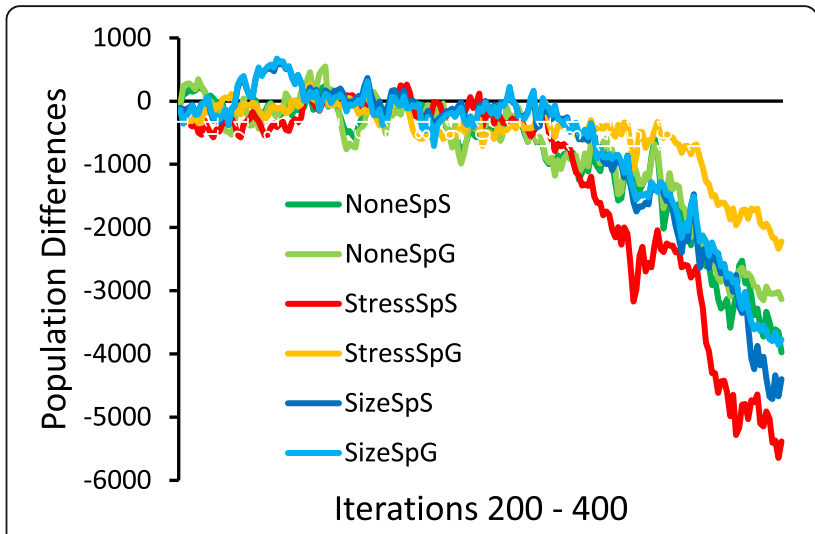

Fig. 2 Absolute difference in sizes from the populations at iteration 200 for the two species in the three feedback models during the iterations of increasing climatic variation (301-400)

increasing variability as it is for SpG. In the size gradient model $\mathrm{SpS}$ is able to advance further into the high stress zone, and without variability SpG has a denser population (fewer empty cells in this area), but with increasing variability SpG is less dense than in any other case.

Before climate change begins in the simulations, differences have developed among the three models (Fig. 3 A,D,G; no feedback, stress gradient, and size gradient). Relative to no feedback, the stress gradient has the ecotone between $\mathrm{SpS}$ and $\mathrm{SpG}$ in about the same place on the grid but the transition zone is longer (Fig. $3 \mathrm{~B}, \mathrm{E}$ ). Additionally, SpG is denser farther upslope. With the size gradient model the transition zone is farther upslope, the area of SpG dominance is about the same, but its density is similar to the case with no feedback (less than for the stress gradient) (Fig. $3 \mathrm{C}, \mathrm{I}$ ).

\section{Discussion}

The relative size gradient model assumes that neighbor interactions affecting a focal individual reach a positive peak when the neighbors are somewhat larger, but become negative when much larger. It is a more mechanistic portrayal of feedbacks on an environmental gradient than the SGH, because of its direct tie between the resulting size and the mechanism of growth. However, in observed effects it is modestly different from simulations based on the SGH. For both models the differences from the no-feedback simulations are qualitatively similar in population responses and spatial patterns.

Table 2 The percent of simulation runs in which a species went extinct ( $N=$ no climatic variability; $V=$ climatic variability)

\begin{tabular}{lllllll}
\hline & NoneN & NoneV & StressN & StressV & SizeN & SizeV \\
\hline Sp1 & 0 & 10 & 3 & 11 & 1 & 12 \\
Sp2 & 1 & 10 & 3 & 12 & 1 & 12 \\
\hline
\end{tabular}




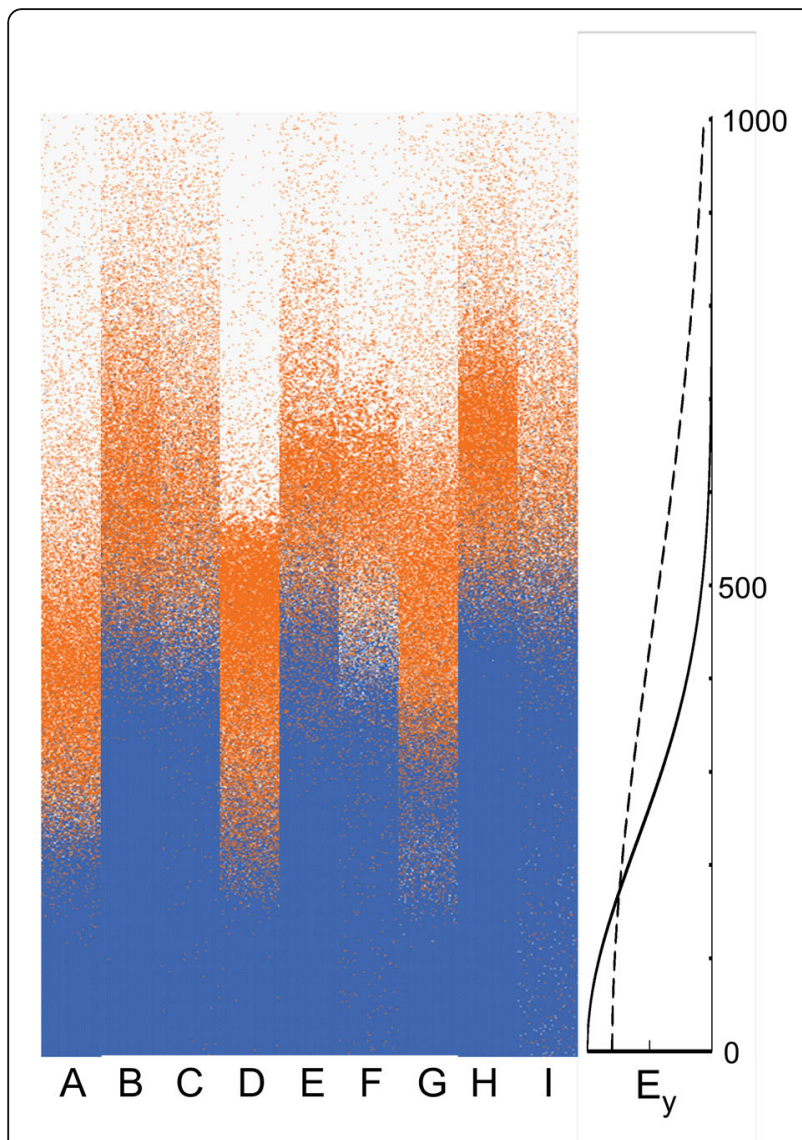

Fig. 3 Comparison of the spatial patterns across the rows of the grid (graph $E_{y}$ corresponds to Fig. 1) produced by the simulations for SpS (blue) and SpG (orange). ABC, DEF, and GEH use no feedbacks or those for the stress and size models, respectively. The patterns are shown prior to beginning climate change in the simulations (iteration 300: A, $D, G$ ) and for after climate change (iteration 400) without increasing variability $(B, E, H)$ and with increasing variability $(C, F, I)$. Base variance has the standard deviation of 0.22

While we had expected that the size gradient model would be less sensitive to increasing climatic variability, sensitivity varied by species. Without any feedback, SpG was more sensitive to increasing variability as climate improved than SpS. Although SpG was able to move upslope in both cases, with increasing variability it did not lead to as many individuals over the entire gradient and so appears to be less dense spatially. With the stress gradient model of feedback, $\mathrm{SpS}$ is more sensitive than SpG. The increasing climatic variability restricts the upslope advance of both species, but to a greater extent for $\mathrm{SpS}$, leaving a less dense ecotone with a preponderance of SpG. The effects switch in the size gradient model. Although the upslope advance is similar with and without increasing climatic variability, in both cases the distribution of SpG is less dense.

The effects of increasing climatic variability depend on interactions. First, although we do not differentiate intraspecific and interspecific interactions in the model, the latter occur. Scramble competition occurs in the ecotone between SpS and SpG regardless of whether the individual interactions are positive or negative. Second, undifferentiated individual interactions can be based on location.

In the stress gradient model, $\mathrm{SpS}$ is confined to the area that experiences competition because the environment is less stressful. As the environment improves, $\mathrm{SpS}$ moves upslope, but still primarily experiences competition among individuals. With increasing climatic variability, improvements in the environment are accompanied by increases in competition so $\mathrm{SpS}$ is not able to take advantage of those positive anomalies. SpG is less affected because it has open area to advance into when the environment improves and it is experiencing modest facilitation which is only increased when the anomalies are negative.

In the size gradient model, SpG is able to take advantage of the improving environment without climatic variability by moving upslope into previously unoccupied area. With increasing variability, the dependence on size buffers its response to the favorable anomalies because there is a biological limit to improvement (the response is never greater than 0.7 ). A negative anomaly that results in mortality is not easily reversed in a subsequent year. Thus the net outcome is highest sensitivity for the scenario we predicted would be lowest.

The mode of interaction does mediate the effects of increasing climatic variability in our model, but not as expected. The size gradient model is more sensitive to increasing climatic variability because of the way that the boundary and interactions between the two species respond. The interaction at the boundary is affected by how species respond to the environmental gradient at the point where they intersect in Fig. 1; cf. [11, 40]. Climatic variability should have the greatest effect on $\mathrm{SpS}$ because its response curve is steeper at the crossover point. This is also where size differences do not buffer responses because the greatest differences in size of neighbors is in the least stressful part of the gradient where individual sizes can be large next to newly recruited neighbors.

The size gradient model introduces a specific feedback mechanism that allows a change from facilitation to competition as the relative sizes of the plants diverge. This model introduces a single functional trait, but from a different perspective than most of the functional traits commonly discussed for plant species. Most work on functional traits is about indicators of functions within individuals [46]. Our perspective comes from the consequences of altering the abiotic environment of a neighborhood. Some commonly used traits, such as canopy height, can have either individual or neighborhood functions, but differentiating the two will be important in assessing their role in plant community response to climate change [8]. 


\section{Conclusions}

The consequences of increasing climatic variability as climate changes depend on the environmental gradient as experienced from the plant's-eye-view, which is affected by interactions with neighbors. At ecotones, this plant's-eye-view determines the transition in dominance from one species or vegetation type to another. In our model this interaction is determined by the differences in slopes of the species response to the environment near their point of intersection as represented in Fig. 1 but further changed by whether neighbor interactions are competitive or facilitative according to the SGH.

Broad representation of species niche based on presence without quantified interactions will be inadequate for assessing their response to climate change. While long recognized [47] but still often ignored, inclusion of plant-plant interactions is necessary to model the transient responses of ecosystems to climate change (along with dispersal and fragmentation). We now see that interactions are even more diverse, more interwoven, and more important. The plant's-eye-view will be necessary to move beyond simplistic species distribution models.

For the specific type of ecotones that motivated this model, alpine treelines, both variability and the nature of stress gradient interactions will be relevant to their response to climate change. For Pinus albicaulis, instances of dieback as modeled here have been observed in California and attributed to water deficit [48]. Inclusion of temporal autocorrelation of climatic variability is warrented. How individuals and species interact is also likely to affect the spatial response of the species to climatic change. Interactions could exacerbate or ameliorate dieback, depending on the climatic parameter that drives the response [37], but general indications of facilitation even in dry environments would support an advance of treeline barring the extremes modeled here or exogenous disturbances (fire, pests, disease) not included (cf. [49]).

\section{Additional file}

Additional file 1: The Netlogo code for the size gradient model. The Netlogo system can be downloaded at https://ccl.northwestern.edu/ netlogo/. (NLOGO $22 \mathrm{~kb}$ )

\section{Abbreviations}

SGH: Stress gradient hypothesis

\section{Acknowledgments}

This material is based upon work while GPM served at the National Science Foundation. Any opinion, findings, and conclusions or recommendations expressed in this material are those of the author and do not necessarily reflect the views of the National Science Foundation.

\section{Availability of data and material}

The Netlogo simulation code is available in the Netlogo community models archive at: https://ccl.northwestern.edu/netlogo/models/community/ index.cgi. Search MalansonReslerTomback.

\section{Authors' contributions}

All authors contributed substantially to the ideas presented in this paper. GPM led the model development phase. GPM and LRM led the writing, and all edited the manuscript. All authors read and approved the final manuscript.

\section{Competing interests}

The authors declare that they have no competing interests.

Consent for publication

NA.

Ethics approval and consent to participate

NA.

\section{Author details}

1Department of Geographical \& Sustainability Sciences, University of lowa, lowa City, IA 52242, USA. ²Department of Geography, Virginia Tech, Blacksburg, VA 24061, USA. ${ }^{3}$ Department of Integrative Biology, CB 171 University of Colorado Denver, P.O. Box 173364, Denver, CO 80217, USA.

Received: 27 September 2016 Accepted: 4 January 2017

Published online: 19 January 2017

\section{References}

1. Easterling DR, Meehl GA, Parmesan C, Changnon SA, Karl TR, Mearns LO. Climate extremes: observations, modeling, and impacts. Science. 2000;289: 2068-74.

2. Helmuth B, Russell BD, Connell SD, Dong Y, Harley CDG, Lima FP, et al. Beyond long-term averages: making biological sense of a rapidly changing world. Clim Change Resp. 2014;1:6. doi:10.1186/s40665-014-0006-0.

3. Vasseur DA, DeLong JP, Gilbert B, Greig HS, Harley CDG, McCann KS, et al. Increased temperature variation poses a greater risk to species than climate warming. Proc Roy Soc B. 2014;281:20132612.

4. Smith MD. The ecological role of climate extremes: current understanding and future prospects. J Ecol. 2011:99:651-5.

5. Reyer CPO, Leuzinger S, Rammig A, Wolf A, Bartholomeus RP, Bonfante A, et al. A plant's perspective of extremes: terrestrial plant responses to changing climatic variability. Glob Change Biol. 2013;19:75-89.

6. Chesson P. Mechanisms of maintenance of species diversity. Ann Rev Ecol Syst. 2000:31:343-66

7. Filazzola A, Lortie CJ. A systematic review and conceptual framework for mechanistic pathways of nurse plants. Glob Ecol Biogeogr. 2014:23:1335-45.

8. Heikkinen RK, Luoto M, Virkkala R. Does seasonal fine-tuning of climatic variables improve the performance of bioclimatic envelope models for migratory birds? Divers Distr. 2006;12:502-10.

9. Kayler ZE, De Boeck HJ, Fatichi S, Grunzweig JM, Merbold L, Beier C, et al. Experiments to confront the environmental extremes of climate change. Front Ecol Env. 2015;13:219-25.

10. Gherardi LA, Sala OE. Enhanced interannual precipitation variability increases plant functional diversity that in turn ameliorates negative impact on productivity. Ecol Lett. 2015;18:1293-300.

11. Malanson GP. Interactions and constraints in model species response to environmental heteroscedasticity. 2016. available online at ir.uiowa.edu.

12. Kupfer JA, Cairns DM. The suitability of montane ecotones as indicators of global climatic change. Prog Phys Geogr. 1996;20:253-72.

13. Malanson GP, Resler LM, Bader MY, Holtmeier F-K, Weiss DJ, Butler DR Mountain treelines: a roadmap for research orientation. Arct Antarct Alp Res. 2011:43:167-77.

14. Wilson JB, Agnew ADQ. Positive feedback switches in plant communities. Adv Ecol Res. 1992:23:263-336.

15. Alftine KJ, Malanson GP. Directional positive feedback and pattern at an alpine tree line. J Veg Sci. 2004;15:3-12.

16. Bader MY, van Geloof I, Rietkerk M. High solar radiation hinders tree regeneration above the alpine treeline in northern Ecuador. Plant Ecol. 2007;191:33-45. 
17. Bourgeron PS, Humphries HC, Liptzin D, Seastedt TR. The forest-alpine ecotone: a multi-scale approach to spatial and temporal dynamics of treeline change at Niwot Ridge. Plant Ecol Divers. 2015;8:763-79.

18. Wheeler JA, Hermanutz L, Marino PM. Feathermoss seedbeds facilitate black spruce seedling recruitment in the forest-tundra ecotone (Labrador, Canada). Oikos. 2011;120:1263-71.

19. Michalet R, Le Bagousse-Pinguet $Y$, Maalouf J-P, Lortie CJ. Two alternatives to the stress-gradient hypothesis at the edge of life: the collapse of facilitation and the switch from facilitation to competition. J Veg Sci. 2014 25:609-13.

20. De Dios VR, Weltzin JF, Sun W, Huxman TE, Williams DG. Transitions from grassland to savanna under drought through passive facilitation by grasses. J Veg Sci. 2014;25:937-46.

21. Dial RJ, Smeltz TS, Sullivan PF, Rinas CL, Timm K, Geck JE, Tobin SC, Golden TS, Berg EC. Shrubline but not treeline advance matches climate velocity in montane ecosystems of south-central Alaska. Glob Change Biol. 2016;22 1841-56.

22. Camarero JJ, Gutierrez E. Pace and pattern of recent treeline dynamics: response of ecotones to climatic variability in the Spanish Pyrenees. Clim Change. 2004;63:181-200.

23. Holtmeier F-K, Broll G. Wind as an ecological agent at treelines in North America the Alps and the European subarctic. Phys Geogr. 2010;31:203-33.

24. Germino MJ, Smith WK. Sky exposure crown architecture and lowtemperature photoinhibition in conifer seedlings at alpine treeline. Plant Cell Environ. 1999;22:407-15.

25. Körner $C$. A reassessment of high elevation treeline positions and their explanation. Oecologia. 1998;115:445-59.

26. Pyatt JC, Tomback DF, Blakeslee SC, Wunder MB, Resler LM, Boggs LA, et al. The importance of conifers for facilitation at treeline: comparing biophysical characteristics of leeward microsites in whitebark pine communities. Arct Antarct Alp Res. 2016;48:427-44.

27. Liang EY, Wang YF, Piao SL, Lu XM, Camarero JJ, Zhu HF, et al. Species interactions slow warming-induced upward shifts of treelines on the Tibetan Plateau. Proc Natl Acad Sci U S A. 2016;113:4380-5.

28. Körner C. When it gets cold, plant size matters - a comment on treeline. J Veg Sci. 2016:27:6-7.

29. Malanson GP, Resler LM. Neighborhood functions alter unbalanced facilitation on a stress gradient. J Theor Biol. 2015;365:76-83.

30. Bertness MD, Callaway R. Positive interactions in communities. Trends Ecol Evol. 1994:9:191-3.

31. Maestre FT, Callaway RM, Valladares F, Lortie CJ. Refining the stress-gradient hypothesis for competition and facilitation in plant communities. J Ecol. 2009;97:199-205.

32. Soliveres S, Maestre FT. Plant-plant interactions, environmental gradients and plant diversity: A global synthesis of community level studies. Persp Plant Ecol Evol Syst. 2014;16:154-63.

33. Ettinger AK, HilleRisLambers J. Climate isn't everything:competitive interactions and variation by life stage will also affect range shifts in a warming world. Am J Bot. 2013;190:344-55.

34. Drake JM. Population effects of increased climate variation. Proc R Soc B. 2005:272:1823-7.

35. Harper JL. Population biology of plants. London: Academic; 1977.

36. Moran EV, Ormond RA. Simulating the interacting effects of intraspecific variation, disturbance, and competition on climate-driven range shifts in trees. PLoS One. 2015;10(11):e0142369. doi:10.1371/journal.pone.0142369.

37. Resler LM, Shao Y, Tomback DF, Malanson GP, Smith-McKenna EK Predicting the functional role of whitebark pine (Pinus albicaulis) at alpine treelines: Model accuracy and variable importance. Ann Assoc Am Geogr. 2014:104:703-22

38. Antos JA, Parish R, Nigh GD. Effects of neighbors on crown length of Abies lasiocarpa and Picea engelmannii in two old-growth stands in British Columbia. Can J For Res. 2010;40:638-47.

39. Malanson GP, Resler LM. A relative size gradient hypothesis for alpine treeline ecotones. J Mtn Sci. 2016;13:1154-61.

40. Wilensky U. NetLogo. http://ccl.northwestern.edu/netlogo/. Center for Connected Learning and Computer-Based Modeling. Evanston, IL: Northwestern University; 1999.

41. Malanson GP. Diversity differs among three variations of the stress gradients hypothesis in two representations of niche space. J Theor Biol. 2015;384:121-30.
42. Smith-McKenna E, Malanson GP, Resler LM, Carstensen LW, Prisley SP, Tomback DF. Cascading effects of feedbacks, disease, and climate change on alpine treeline dynamics. Environ Model Software. 2014;62:85-96.

43. Bekker MF. Positive feedback between tree establishment and patterns of subalpine forest advancement, Glacier National Park, Montana, USA. Arc Antarct Alp Res. 2005;37:97-107.

44. Alftine KJ, Malanson GP, Fagre DB. Feedback-driven response to multidecadal clmatic variability at an alpine forest-tundra ecotone. Phys Geogr. 2003:24:520-35.

45. Malanson GP, Butler DR, Cairns DM, Welsh TE, Resler LM. Variability in a soil depth indicator in alpine tundra. Catena. 2002:49:203-15.

46. Butterfiled BJ, Callaway RM. A functional comparative approach to facilitation and its context dependence. Funct Ecol. 2013;27:907-17.

47. Malanson GP. Comment on modeling ecological response to climatic change. Clim Change. 1993:23:95-109.

48. Millar Cl, Westfall RD, Delany DL, Bokach MJ, Flint AL, Flint LE. Forest mortality in high-elevation whitebark pine (Pinus albicaulis) forests of eastern California, USA; influence of environmental context, bark beetles, climatic water deficit, and warming. Can J For Res. 2012;42:749-65.

49. Tomback DF, Blakeslee SC, Wagner AC, Wunder MB, Resler LM, Pyatt JC, Diaz S. Whitebark pine facilitation at treeline: potential interactions for disruption by an invasive pathogen. Ecol Evol. 2016:6:5144-57.

\section{Submit your next manuscript to BioMed Central and we will help you at every step:}

- We accept pre-submission inquiries

- Our selector tool helps you to find the most relevant journal

- We provide round the clock customer support

- Convenient online submission

- Thorough peer review

- Inclusion in PubMed and all major indexing services

- Maximum visibility for your research

Submit your manuscript at www.biomedcentral.com/submit 